\title{
Bitcoin Prediction using Rain Optimization Algorithm
}

\author{
Mustufa Haider Abidi \\ Advanced Manufacturing Institute, \\ King Saud University, Riyadh, Saudi Arabia \\ mabidi@ksu.edu.sa
}

\begin{abstract}
The development in stock market prediction is represented as a significant role and it received immense interest as predicting stock prices effectively which cause striking profits by creating appropriate decisions. Stock market prediction is the most important confronts on account of non-stationary, blaring, and chaotic data. Hence, prediction turns out to be demanding between investors to invest the money in order to make earnings. At first, to blockchain Network Bridge the blockchain network is subjected from that bitcoin data is obtained which is pursued with the prediction of bitcoin. The developed Raindrop optimization method based on Deep LSTM is used for the prediction of Bitcoin. Initially, based on the Rate of Change Rate (ROCR), Average True Range (ATR), Double Exponential Moving Average (DEMA), Simple Moving Average (SMA), and Moving Average Convergence Divergence (MACD) flow strength indicators are extracted from the blockchain data. On the basis of the extracted features, the prediction is performed exploiting Raindrop optimization algorithm-based Deep LSTM, which is a combination of Raindrop optimization algorithm with Deep LSTM. Subsequently, the Raindrop optimization algorithm is used to determine the optimal weights in Deep LSTM. The simulation of the developed algorithm is done from the explicitly accessible dataset. The examination of technique regarding MAE and RMSE shows that the developed method attained a minimal Mean Absolute Error (MAE) and the minimal Root Mean Squared Error (RMSE) correspondingly.
\end{abstract}

Keywords: Stock Market, Bitcoin, Prediction, BlockChain, Optimization Algorithm

\begin{tabular}{ll} 
Nomenclature & \\
\hline Abbreviation & Description \\
\hline ROCR & Rate of Change \\
ARMA & Autoregressive Moving Average \\
FLANN & Functional Link Artificial Neural Network \\
SVM & Support Vector Machine \\
SMA & Simple Moving Average \\
ML & Machine Learning \\
ANN & Artificial Neural Network \\
ATR & Average True Range \\
ARIMA & Autoregressive Integrated Moving Average \\
DEMA & Double exponential moving average \\
RMSE & Root Mean Square Error \\
ECDSA & Elliptic Curve Digital Signature Algorithm \\
MAE & Mean Absolute Error \\
MACD & Moving Average Convergence Divergence \\
DNN & Deep Neural Network \\
FF & Firefly \\
KAF & Kernel Adaptive Filtering \\
QoS & Quality of Service \\
\hline
\end{tabular}

\section{Introduction}

Stock returns are incessantly produced by diverse and detach data sources, like corporations, banks, as individuals. Hence, in contrast with the conventional classification and regression issues, forecasting these time-series needs deliberation of an incessant series of data records, the interdependency among the stock markets. Using nonlinearities and high noise, the stock returns are classified that inflict extra confronts to attaining reliable prediction. 
In stock market trading systems an additional application for the prediction of the stock market can be established, which typically comprise various modules for risk analysis, prediction, and trading schemes. The objective of a trading element is to generate a stock portfolio that increases on the whole revisit concerning stock risk in that portfolio. Nevertheless, a prediction element concentrates on subissue to predict markets future which is a precious bit of the pack of information in the stock trading process. Hence, module performance amount the complete trading system, which is prejudiced significantly by the prediction quality that occurs in the prediction module. Actually, without a consistent prediction, it is approximately not possible to encompass an outstanding trading system.

In a different way, several researchers have made an attempt to experimentally show to be false the $\mathrm{EMH}$, and observational proofs, possess outlined that stock market is predicted to a few amount. Several conventional methods were introduced by researchers in forecasting field price movements of stock like ARMA, ARIMA, etc., however, these techniques are having few restrictions, they are not able to hold nonlinear relationship subsist in time series data as they create the supposition of a linear form of model construction. Moreover, they are assumed to have constant variance, where, financial time series data are noisy by nature. Afterward, problems concerning the subsistence of nonlinearity have been resolved in time-series data by the technique of numerous ML approaches like ANN, FLANN, and SVM so forth. In the past decades, various researchers encompass discovered the prediction capability of $\mathrm{NN}$ for the prediction of the financial market.

The most important aim of this article is to recommend a technique for bitcoin prediction. Therefore, the Raindrop optimization algorithm-based Deep LSTM is presented which is the combination of Raindrop optimization algorithm conception in the Deep LSTM method to carry out the bitcoin prediction. Conversely, Deep LSTM is trained optimally by exploiting an optimization algorithm.

\section{Literature Survey}

In 2020, Sergio Garcia-Vega et al [1], presented exploits of a two-stage technique, exploiting KAF in a stock market interdependence technique to predict stock returns sequentially. The improved KAF plus market interdependence model has experimented on 24 diverse stocks from the most important economies. The improved technique attains a superior Sharpe ratio while evaluated with KAF-based techniques, short long-term memory, and autoregressive-based techniques. In 2019 Ehsan Hoseinzade and Saman Haratizadeh [2] suggested a CNN-based model, which was used on a data collection from a diversity of sources, involving diverse markets, to feature extraction to predict the future of those markets. The proposed model was used to predict the subsequent day's direction of movement on basis of the several sets of initial variables. In 2020, Jiawei Long et al [3], presented a DNN model exploiting the public market information and desensitized transaction records to predict stock price trends. Taking into consideration of the correlation among stocks, the proposed technique uses the knowledge graph and graph embeddings methods. In 2019, Xiaodong Li et al [4], worked on a stock prediction system and developed a method that indicates numerical price data using technical indicators by analyzing technically, and the sentiment vectors, was used to indicate textual news articles through sentiment analysis. To learn the sequential information the layered deep learning model was set up within the market snapshot series. In 2019, Smruti Rekha Das et al [5], worked on the feature optimization capacity of FF with an evolutionary model by social and biochemical concepts of FF approach, besides with the chosen process of objective value in evolutionary notion. The developed technique performance was evaluated exploiting four diverse stock market datasets. In 2017, Eunsuk Chong et al [6], attempted to present an objective assessment and comprehensive of both benefits and disadvantages of deep learning methods for stock market prediction and analysis.

\section{BlockChain Based Approach Using Proposed Model}

The bitcoin market prediction receives more benefits in evaluated with normal stock markets. Bitcoin is not on the basis of events regarding intervening business or governments and price is decided to exploit coins shortage. "The Bitcoins are mainly based upon a humans' trust in coin, predicting if it goes up or down subsequent day". Fig. 1 demonstrates the architecture model of the developed algorithm.

Initially, the blockchain network is fed to blockchain Network Bridge, whereas 4 processes happen, that comprises block chain, Sensor Bridge, blockchain wallet, and Machine Bridge. The outcome attained from the blockchain network bridge is bitcoin data that is subjected to the prediction of bitcoin. Bitcoin prediction is carried out exploiting Raindrop Optimization algorithm-based Deep LSTM that is a combination of Raindrop Optimization algorithm with Deep LSTM. 


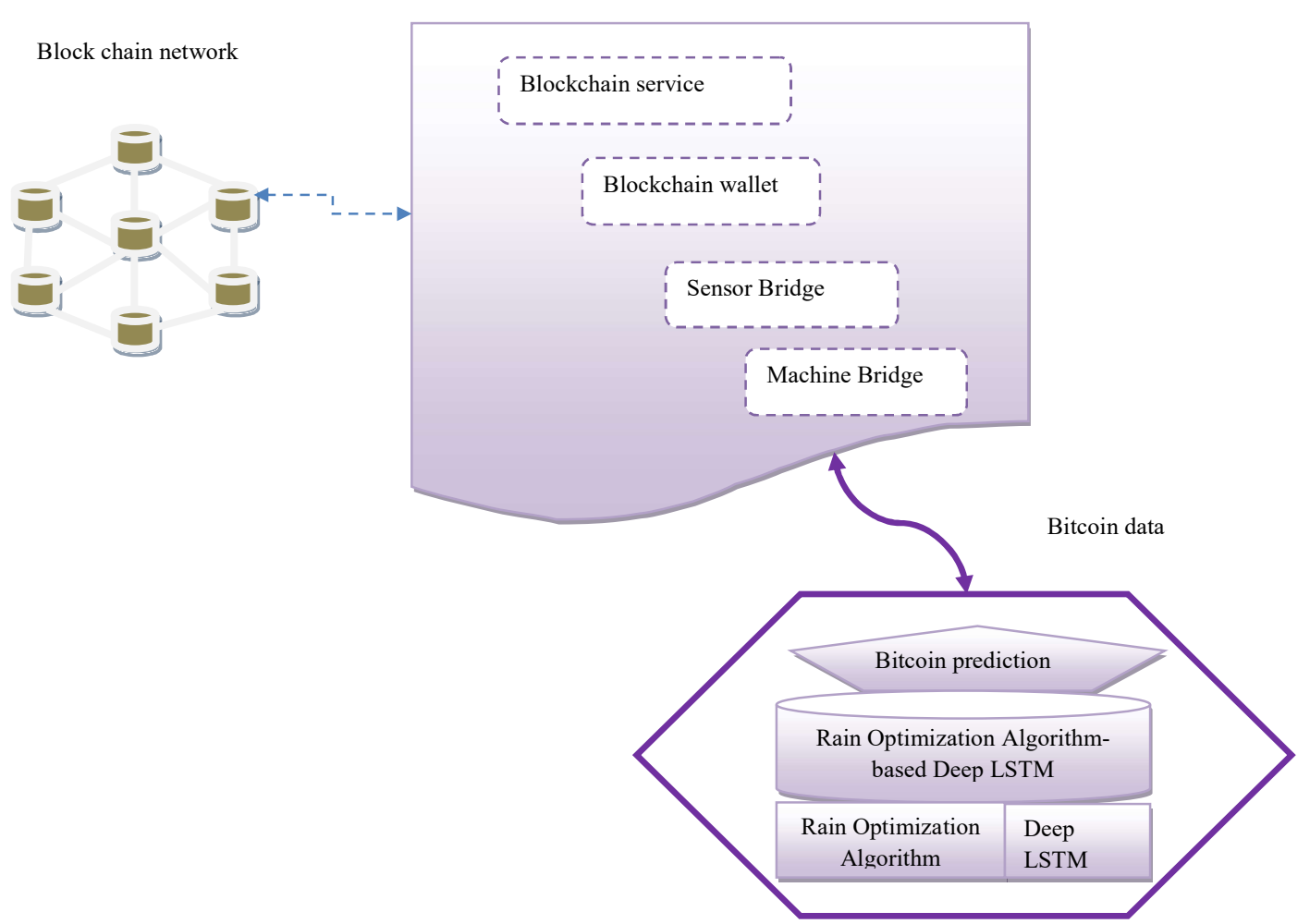

Fig. 1. Schematic model of the developed method

\subsection{Blockchain Structure}

By cryptocurrency, Bitcoin is described which is used for investment procedures or digital payment. Bitcoin is classified into the public chain, private chain, and federated chains. Bitcoin is decentralized; it represents that bitcoin data is not controlled by anyone. Transactions are performed using Bitcoins which is very easy however they are not combined with any nation. Bitcoin exchanges are used to perform investments using several market regions. This permits people to sell or buy Bitcoins on the basis of several currencies. The major exploited Bitcoin exchange is Mt Gox. Moreover, in a digital wallet, Bitcoins are stored that is similar to a bank account in a virtual manner. In Blockchain, all transaction records, and timestamp data, are saved. In a blockchain, every record is referred to as a block that comprises a pointer to a previous data block. Then, the encryption is performed in blockchain data. At the time of transactions, the name of users is not exhibited; however, their wallet ID is done publicly.

a) Blockchain Network Bridge: The single node is subjected to blockchain Network Bridge from blockchain that engages the subsequent processes, such as blockchain service, Sensor Bridge, blockchain wallet, and Machine Bridge as defined as follows:

b) Blockchain service: The fundamental data-grid feature is to offer astonishing QoS. Exploiting the viewpoint of industrial growth, the most important industrial sectors exploited in blockchain are information technology and software services. The majority of blockchain technology providers might employ information technology services and associated software products, namely system integration.

c) Blockchain wallet: A wallet comprises private keys, one is allocated to every account. On the basis of the master key, the private key is encrypted, which is known as an arbitrary key. Additionally, it is encrypted by superior encryption standards. The incorporation of the private key, and public key, is used to produce a digital signature to transact from peer-to-peer. The ECDSA is exploited by Bitcoin for secret sharing, encryption, and threshold cryptography

d) Sensor bridge: For sensing, it needs actuators and sensors. The sensor is a device which is exploited to detect and respond to electrical or optical signals; it transforms physical parameter, such as blood pressure, humidity, temperature, speed, etc to a signal which is deliberate electrically. An actuator is a machine exploited to control or moves a system. Subsequent to sensing, from the machine bridge bitcoin data is attained. 


\subsection{Bitcoin Prediction Exploiting Developed Raindrop Optimization Technique -based Deep LSTM}

For bitcoin detection the developed Raindrop optimization algorithm-based Deep LSTM is presented in this section. Bitcoin is the world's most important cryptocurrency that permits users to make transactions safe and anonymous over the Internet. Therefore, this paper develops a Raindrop optimization algorithm-based Deep LSTM. The developed bitcoin prediction method is categorized into 2 steps such as extraction of flow strength prediction and indicators. Initially, flow strength indicators are extracted by exploiting the DEMA, ATR, ROCR, SMA, and MACD from blockchain data. On the basis of the extracting features, the prediction is performed exploiting developed Raindrop optimization algorithm -based Deep LSTM that is an integration of Raindrop optimization algorithm and Deep LSTM. In the developed Raindrop optimization algorithm -based Deep LSTM, is exploited for best-chosen weights in Deep LSTM.

\subsection{DEEP LSTM Architecture Model}

From the current promising networks for bitcoin prediction, a fully linked deep LSTM network is modeled. Fig 2 shows the deep LSTM model. It comprises three bidirectional LSTM layers, and two feedforward layers, softmax layer for predictions. Here, exploration is subjected to associations offering to subsequent LSTM layers to learn co-incidences of joints or features. LSTM dropout is fed to the preceding LSTM layer for efficient learning. Moreover, every LSTM layer uses bidirectional LSTM to distinguish forward and backward LSTM neurons. At each step, a vector is considered as input to the system indicating 3D locations of joints of the skeleton in a frame. The encoding LSTM is used to compress input into hidden states to forecast bitcoin prediction. Each LSTM network comprises memory units that comprise the cell and gates and computation of output from individual modules of the memory unit is shown. The gates, and memory cell, play an important role in managing flow of information, additionally, an encoding-forecasting model of DeepLSTM [7] make a sure forecast on basis of the spatiotemporal series. The memory cell, and gates, restore ordinal neurons in other deep learning models hence memory cell state of DeepLSTM is updated on basis of the new input, not remember superfluous contents, and output the consequence. The aforesaid features of DeepLSTM produce it appropriate for bitcoin prediction. The computation of input and output gates from the memory unit is described as follows:

The DeepLSTM is used to hold the memory of precedent. From the input gate, the output is devised as,

$$
\mathrm{P}_{\tau}=\sigma\left(\omega_{\mathrm{P}}^{\mathrm{I}} * \mathrm{I}_{\tau}+\omega_{\mathrm{P}}^{\mathrm{H}} * \mathrm{H}_{\tau-1}+\omega_{\mathrm{P}}^{\mathrm{O}} \circ \mathrm{O}_{\tau-1}+\beta^{\mathrm{P}}\right)
$$

In eq. (1), $I_{\tau}$ indicates input vector, $\omega_{P}^{I}$ indicates weight among the input layer and the gate. $\sigma$ indicates gate activation function that is the majority frequently sigmoid function. $\omega_{\mathrm{P}}^{\mathrm{H}}$ indicates the weight among input and memory output layer, and $\omega_{\mathrm{P}}^{\mathrm{O}}$ indicates weight vector among input and cell output. $\mathrm{H}_{\tau-1}$ and $\mathrm{O}_{\tau-1}$ indicates the preceding output of the cell and the memory unit. The input layer bias is indicated as, $\beta^{\mathrm{P}}$. The Convolutional operator is indicated as, and $\circ$ indicates element-wise multiplication. The outcome from forget gate is calculated as, *

$$
\mathrm{Q}_{\tau}=\sigma\left(\omega_{\mathrm{Q}}^{\mathrm{I}} * \mathrm{I}_{\tau}+\omega_{\mathrm{Q}}^{\mathrm{H}} * \mathrm{H}_{\tau-1}+\omega_{\mathrm{Q}}^{\mathrm{O}} \circ \mathrm{O}_{\tau-1}+\beta^{\mathrm{Q}}\right)
$$

In eq. (2), $\omega_{Q}^{\mathrm{I}}$ indicates the weight among the input layer and forget gate, $\omega_{Q}^{\mathrm{H}}$ indicates the weight which links the output gate and the memory unit of the preceding layer, and $\omega_{Q}^{0}$ indicates the weight among the output gate and cell. $\beta^{Q}$ indicates the bias in line with the forget gate. From the output gate, the result is devised as,

$$
R_{\tau}=\sigma\left(\omega_{R}^{\mathrm{I}} * \mathrm{I}_{\tau}+\omega_{\mathrm{R}}^{\mathrm{H}} * \mathrm{H}_{\tau-1}+\omega_{\mathrm{R}}^{\mathrm{O}} \circ \mathrm{O}_{\tau}+\beta^{\mathrm{R}}\right)
$$

In eq. (3), $\omega_{\mathrm{R}}^{\mathrm{H}}$ indicates weight among the output gate and the memory unit, $\omega_{\mathrm{R}}^{\mathrm{I}}$ indicates weight which connects the output gate and the input layer and $\omega_{\mathrm{R}}^{\mathrm{O}}$ indicates the weight among the output gate and the cell. Consider output gate bias is indicated as, $\beta^{R}$. The output of the impermanent cell state is devised on basis of the activation function of weights in proportion to cell as below:

$$
\tilde{\mathrm{O}}_{\tau}=\tanh \left(\omega_{\mathrm{C}}^{\mathrm{I}} * \mathrm{I}_{\tau}+\omega_{\mathrm{C}}^{\mathrm{H}} * \mathrm{H}_{\tau-1}+\beta^{\mathrm{C}}\right)
$$


In eq. (4), $\omega_{\mathrm{C}}^{\mathrm{I}}$ and $\omega_{\mathrm{C}}^{\mathrm{H}}$ indicates the weights among cell and input layer, and cell and a memory unit, correspondingly. The cell bias is indicated as, $\beta^{\mathrm{C}}$. The cell output is stated as a summation of impermanent cell state and the difference among memory unit of preceding and present layers which are stated as,

$$
\begin{aligned}
& \mathrm{O}_{\tau}=\mathrm{Q}_{\tau} \circ \mathrm{O}_{\tau-1}+\mathrm{P}_{\tau} \circ \tilde{\mathrm{O}}_{\tau} \\
& \mathrm{O}_{\tau}=\mathrm{Q}_{\tau} \circ \mathrm{O}_{\tau-1}+\mathrm{P}_{\tau} \circ \tanh \left(\omega_{\mathrm{C}}^{\mathrm{I}} * \mathrm{I}_{\tau}+\omega_{\mathrm{C}}^{\mathrm{H}} * \mathrm{H}_{\tau-1}+\beta^{\mathrm{C}}\right)
\end{aligned}
$$

From the memory unit, the output is stated as,

$$
\mathrm{H}_{\tau}=\mathrm{R}_{\tau} \circ \tanh \left(\mathrm{O}_{\tau}\right)
$$

In eq. (7), $H_{\tau}$ states the output of memory block, $R_{\tau}$ states output gate. Hence, the output of the output layer is stated as,

$$
\mathrm{Z}_{\tau}=\phi\left(\omega_{\mathrm{Z}}^{\mathrm{H}} \cdot \mathrm{H}_{\tau}+\beta^{\mathrm{Z}}\right)
$$

\subsection{Rain Optimization Algorithm (ROA)}

In eq. (8), $Z_{\tau}$ indicates the output vector and $\omega_{Z}^{H}$ indicates the weight of the output vector and the memory unit. The output layer bias is indicated as, $\beta^{Z}$

Hence, from the output layer, the output of every LSTM is forwarded to the ensuing LSTM, lastly, the redundant output from LSTMs in the encoding layer is subjected to the forecasting layer for concluding prediction. Hence, the weights, and Deep LSTM bias, are indicated as, $\omega \in\left\{\omega_{\mathrm{Z}}^{\mathrm{H}}, \omega_{\mathrm{C}}^{\mathrm{I}}, \omega_{\mathrm{C}}^{\mathrm{H}}, \omega_{\mathrm{R}}^{\mathrm{I}}, \omega_{\mathrm{R}}^{\mathrm{H}}, \omega_{\mathrm{R}}^{\mathrm{O}}, \omega_{\mathrm{Q}}^{\mathrm{I}}, \omega_{\mathrm{Q}}^{\mathrm{H}}, \omega_{\mathrm{Q}}^{\mathrm{O}}, \omega_{\mathrm{P}}^{\mathrm{I}}, \omega_{\mathrm{P}}^{\mathrm{H}}, \omega_{\mathrm{P}}^{\mathrm{O}}\right\}$ and $\beta \in\left\{\beta^{\mathrm{C}}, \beta^{\mathrm{Q}}, \beta^{\mathrm{R}}, \beta^{\mathrm{P}}\right\}$. The weights tuning $\omega$ and biases $\beta$ of the Deep LSTM are decided optimally exploiting the developed technique.

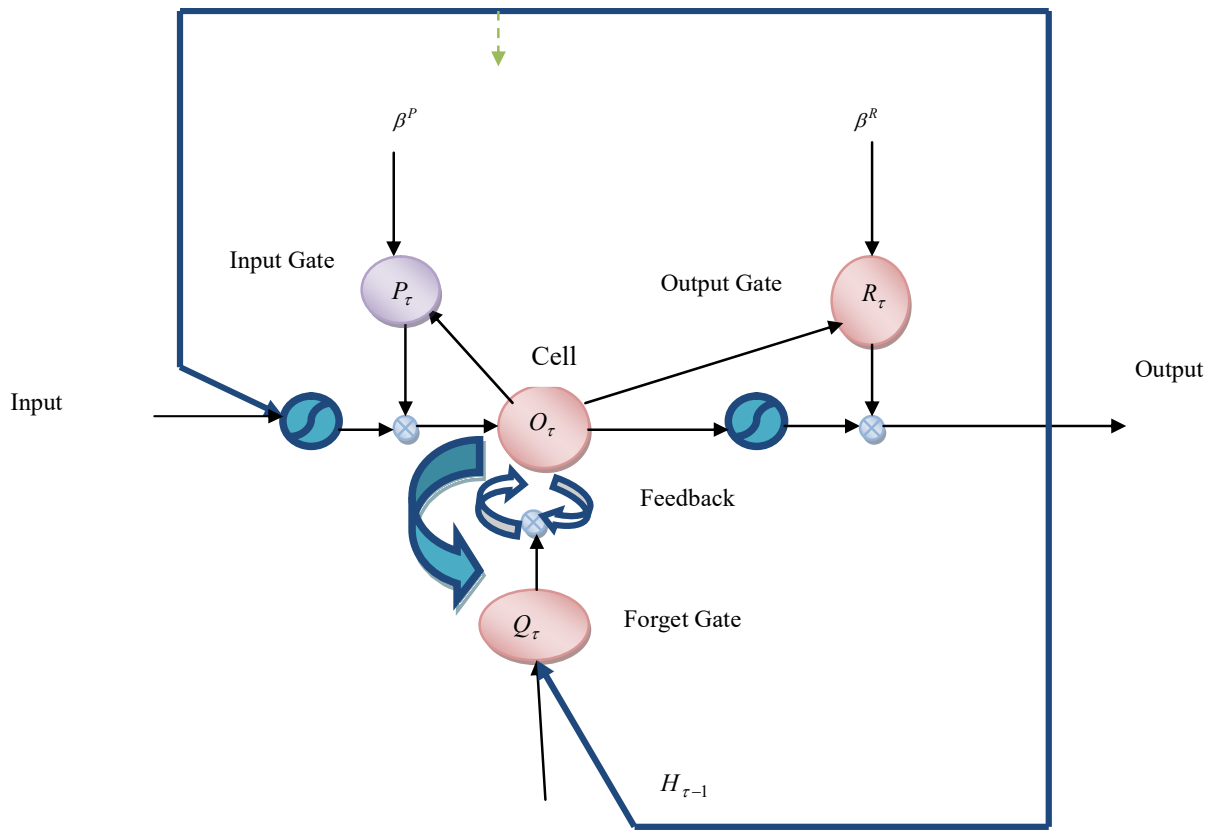

Fig. 2. Network model of the Deep LSTM

In ROA every solution of issue can be designed by a raindrop [9]. Hence at the initial step, the upper, and lower limit of variable one, will be analyzed as these limits can be decided by droplet radius. At the subsequent step, 2 endpoints of variable 2 can be examined and this is sustained till the concluding variable. In this phase, the cost of the initial droplet can be updated by moving it downward [8]. This is not the final action for this droplet, when the cost function is minimizing, it will move downward in a similar direction. For all droplets this action will be done, subsequently, the cost and location of all droplets will be allocated.

For each droplet the radius will be altered in 2 ways:

If two droplets using radius $r_{1}$ and $r_{2}$ are nearer to each other which has a general area with each other; they can link to create a bigger droplet of radius $\mathrm{R}: \mathrm{u}$ 
Bitcoin Prediction using Rain Optimization Algorithm

$$
R=\left(r_{1}^{n}+r_{2}^{n}\right)^{1 / n}
$$

In eq. (9), $\mathrm{n}$ indicates a number of variables in each droplet.

If a droplet with radius $r_{1}$ does not move, which depends on the properties of soil, that is exhibited by $\alpha$, few volume percentages of it can be adsorbed.

$$
R=\left(\alpha r_{1}^{n}\right)^{1 / n}
$$

Actually, $\alpha$ exhibits the percentage of droplet volume that can be absorbed in each iteration and is an amount between 0 to 100 percent. It can describe the least amount for droplets radius $r_{\text {min }}$, whereas droplets with a lesser radius of that $r_{\text {min }}$ will evade. Since it can be considered, the population number could be minimized subsequent to a few iterations and higher droplets will be adopted with a higher domain of examinations. By raising the domain of examination for each drop, the local searching capability of drops is raised proportionally to the droplet's diameter. Hence by raising the number of iteration, weak droplets with a minimum domain of examination evade or link to stronger drops with a superior domain of examination and the initial population will reduce intensively caused by raising the speed of discovering the appropriate answer(s).

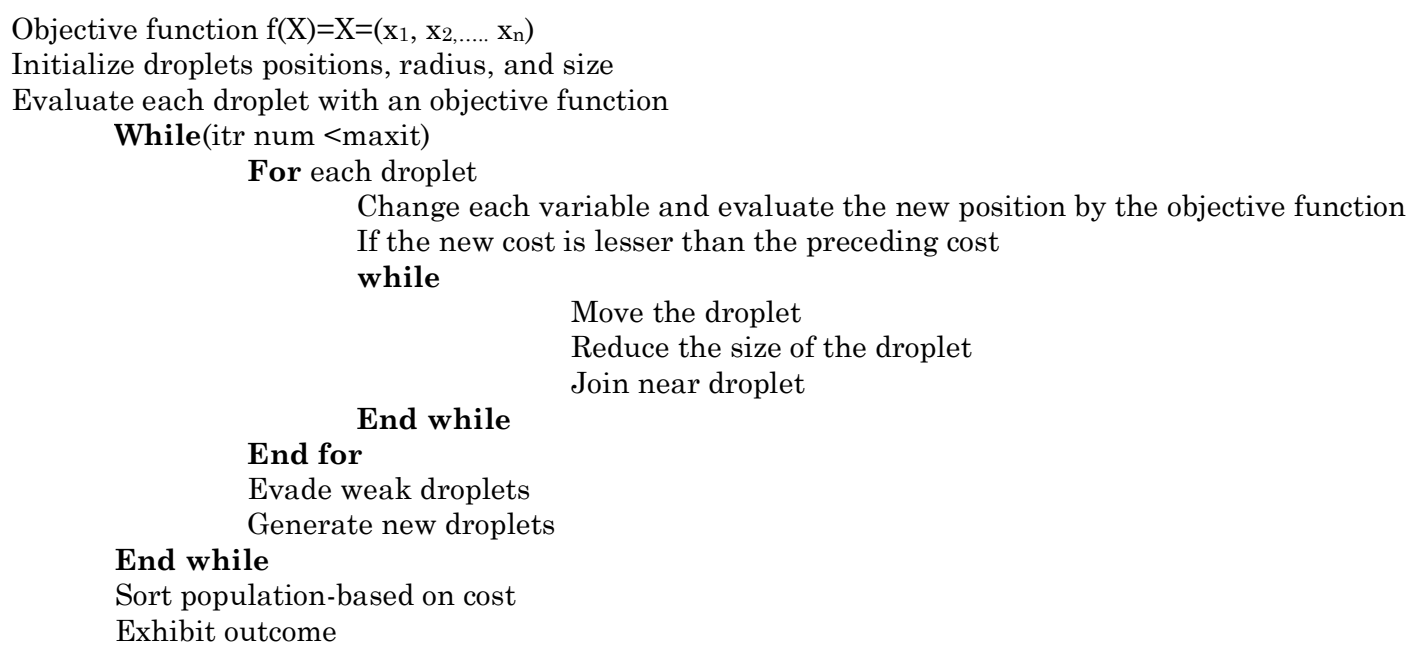

End for

End while

Join near droplet

End while

Evade weak droplets

Generate new droplets

Sort population-based on cost

Exhibit outcome

\section{Result and Discussions}

In this section, the result analysis of the developed bitcoin prediction exploiting Rain Optimization Algorithm-Deep LSTM was shown with an effectual evaluative analysis for showing the effectiveness of the developed technique.

The analysis of the developed model was carried out by exploiting two measures, like MAE, and RMSE.

It is evident from fig 2 and 3 that the average PSNR and SSIM increase with the increasing values of t and the image sizes. Even though the existing methods contributed individually with the effective performance, the proposed method outperforms the existing methods with a good value for PSNR and SSIM, which are the measures that define the quality of the embedded image.

Table 1: Performance analysis of proposed method on dataset 1

\begin{tabular}{lll}
\hline Techniques & RMSE & MAE \\
\hline Deep LSTM & 17.567 & 11.131 \\
NARX & 33.234 & 32.231 \\
Autoregression & 22.323 & 11.431 \\
Proposed method & 2.876 & 0.8824 \\
\hline
\end{tabular}

Table 2: Performance analysis of proposed method on dataset 2

\begin{tabular}{lll}
\hline Techniques & RMSE & MAE \\
\hline Deep LSTM & 0.322 & 8.233 \\
NARX & 0.333 & 0.899 \\
Auto regression & 0.445 & 1.234 \\
Proposed method & 0.122 & 0.6833 \\
\hline
\end{tabular}


Tables 1 and 2 delineates the performance analysis of the proposed and conventional model based on MAE and RMSE for both data set 1 and 2. As shown in Tables 1 and 2, the proposed model has comparatively superior performance while comparing with the conventional methods concerning the MAE, and RMSE respectively.

\section{Conclusion}

In this work, the bitcoin prediction by exploiting the developed Raindrop optimization algorithm based on Deep LSTM was presented. Initially, to the blockchain network bridge, the blockchain network is fed to the generation of the extra block in the blockchain. In the blockchain network, the four blocks are Block chain; blockchain wallet, Sensor Bridge, and Machine Bridge. The outcome attained from the blockchain network bridge was bitcoin data. Subsequently, to bitcoin prediction, bitcoin data was subjected. Bitcoin prediction was done by exploiting the developed Raindrop optimization algorithmbased Deep LSTM. Initially, the extraction was carried out based on the blockchain data. Based on the extracted features, by exploiting Raindrop optimization algorithm-based Deep LSTM the prediction was performed, which was the integration of Raindrop optimization algorithm with Deep LSTM. Subsequently, a Raindrop optimization algorithm was used to determine the optimal weights in Deep LSTM. The simulation of the developed technique was done from the openly accessible dataset. The outcomes of the developed technique were evaluated over the conventional methods, and the proposed method outperforms other approaches by attaining minimal MAE, and RMSE value.

\section{Compliance with Ethical Standards}

Conflicts of interest: Authors declared that they have no conflict of interest.

Human participants: The conducted research follows the ethical standards and the authors ensured that they have not conducted any studies with human participants or animals.

\section{References}

[1] Sergio Garcia-VegaXiao-Jun ZengJohn Keane,"Stock returns prediction using kernel adaptive filtering within a stock market interdependence approach",Expert Systems with Applications26 June 2020.

[2] Ehsan HoseinzadeSaman Haratizadeh,"CNNpred: CNN-based stock market prediction using a diverse set of variables", Expert Systems with Applications20 March 2019.

[3] Jiawei LongZhaopeng ChenJiangtao Ren,"An integrated framework of deep learning and knowledge graph for prediction of stock price trend: An application in Chinese stock exchange market",Applied Soft Computing4 March 2020.

[4] Xiaodong LiPangjing WuWenpeng Wang,"Incorporating stock prices and news sentiments for stock market prediction: A case of Hong KongInformation Processing \& Management12 February 2020.

[5] Smruti Rekha DasDebahuti MishraMinakhi Rout,"Stock market prediction using Firefly algorithm with evolutionary framework optimized feature reduction for OSELM method"Expert Systems with Applications: X30 August 2019.

[6] Eunsuk ChongChulwoo HanFrank C. Park," Deep learning networks for stock market analysis and prediction: Methodology, data representations, and case studies Systems with Applications22 April 2017.

[7] Xingjian Shi, Zhourong Chen, Hao Wang, Dit-Yan Yeung, Wai-Kin Wong, and Wang-chun Woo, "Convolutional LSTM Network: A Machine Learning Approach for Precipitation Nowcasting", Computer Vision and Pattern Recognition, 2015.

[8] Aghay Kaboli, S.Hr., J.Selvaraj, N.A.Rahim, Rain-fall optimization algorithm: a population based algorithm for solving constrained optimization problems, Journal of Computational Science.

[9] Ali Reza MoazzeniEhsan Khamehchi,"Rain optimization algorithm (ROA): A new metaheuristic method for drilling optimization solutions",Journal of Petroleum Science and Engineering1 July 2020. 